\title{
Design of Miniaturized Wideband Microwave Absorber Loaded with Lumped Resistance
}

\author{
Junxiang LAN, Xiangyu CAO, Jun GAO, Lili CONG, Siming WANG, Huanhuan YANG \\ Information and Navigation Institute of Air Force Engineering University, Xi'an, Shaanxi, 710077, China \\ junxianglan@126.com,xiangyucaokdy@163.com,gjgj9694@163.com,1183068955@qq.com,15686018665@163.com, \\ jianye8901@126.com
}

Submitted January 13, 2018 / Accepted May 22, 2018

\begin{abstract}
In this paper, a polarization and angular insensitive microwave absorber with miniaturized structure is proposed. This structure integrates lumped resistances into a metallic bow-tie structure, thus achieving wideband absorbing performance. It can be found that the metallic bow-tie structure increases the equivalent capacitance and equivalent inductance, which enables the absorber with an electrically small structure. The presence of substrate and air layer contributes a lot to the broadband absorption in relative low frequency band. The size of the unit is about $0.092 \lambda_{L}$, and the total thickness is only $0.080 \lambda_{L}$ in correspondence to the lowest frequency. Simulated results show that the absorptivity exhibits good stability with respect to different polarized incidence and different incident angles. The absorber exhibits wideband absorptivity above 90\% from $2.3 \mathrm{GHz}$ to $9.6 \mathrm{GHz}$ with a relative absorption bandwidth of about 123\%. The measured results are in good agreement with the simulated one, which demonstrates the absorber capable of good potentiality in electromagnetic (EM) stealth applications.
\end{abstract}

\section{Keywords}

Microwave absorber, wideband, miniaturization, lumped resistance

\section{Introduction}

With the development of EM war and radar detection system, more and more attention is paid to the stealth capability of equipment. Microwave absorber is a kind of artificial composite material. Owing to its reflection suppression, it has been applied in EM stealth applications. In 2008, Landy first proposed perfect absorbing material (PMA) [1]. Compared to the traditional radar absorbing materials, PMA has such advantages as thinner thickness, lighter weight, and perfect absorbing performance and so on. In recent years, PMA has attracted much attention and develop rapidly. Many unique characteristics of PMA were achieved, such as polarization insensitive absorbing [2], [3], multiple band absorbing [4-6], wide incident angle absorbing [7], [8], tunable absorbing [9-11], miniature absorbing [12]. However, the narrow bandwidth restricts wide engineering application of PMA. Therefore, wideband microwave absorber (WBMA) becomes an advanced research hotspot. Meanwhile, many different WBMAs are designed and fabricated. In [13], a novel triple-layer microwave absorber which combines nested circle rings with stacking metal-dielectric layers is designed. The simulation results exhibit perfect absorption which is beyond 90\% from $1.666 \mathrm{THz}$ to $2.562 \mathrm{THz}$ with an absolute bandwidth of $0.896 \mathrm{THz}$. In [14], the structure of the designed WBMA consists of two magnetic sheets layers. And the bandwidth of absorptivity below $-10 \mathrm{~dB}$ is in the range of $4.0 \mathrm{GHz}$ to 18.0 GHz. In [15], a WBMA composed of three layers of square resistive metasurfaces with different dimensions is designed, and experimentally demonstrated to achieve ultra-wideband absorption. The operating bandwidth with absorption over $90 \%$ is from $7.0 \mathrm{GHz}$ to $37.4 \mathrm{GHz}$; the relative absorption bandwidth is about $137 \%$. In [16], lowprofile and broadband absorbers based on capacitive surfaces are proposed. The capacitive surfaces are achieved by the combination of two ring resonators. The WBMA can realize wideband absorption with a relative bandwidth about $117.4 \%$, while the thickness of the WBMA is only about $0.076 \lambda_{\mathrm{L}}$ at the lowest operating frequency.

There are four important parts in the process of designing an absorber, including the absorption bandwidth, absorptivity over the operating band, thickness, and size of the structure. Thus, it is necessary to design a miniaturized absorber with wide operation band and high absorptivity efficiency. In this paper, we report on the design, fabrication, measurement, and analysis of an ultra-wideband absorber based on loading lumped resistances in terms of miniaturization and the absorption bandwidth. The metallic bow-tie structure increases the equivalent capacitance and the equivalent inductance, making the absorber resonant at a lower frequency. The presence of substrate and air layer contributes a lot to the broadband absorption in relative low frequency band. Lumped resistances are introduced to adjust impedance matching, and then to further broaden the absorption bandwidth. 


\section{Design and Simulation Analysis of WBMA}

\subsection{Structures of the WBMA Unit Cell}

The unit of WBMA is shown in Fig. 1. The periodicity of unit $p$ is $12 \mathrm{~mm}$ with an electrical dimension of $0.092 \lambda_{\mathrm{L}}$ with respect to the lowest frequency in operation. The total thickness is $10.5 \mathrm{~mm}$, and it is only about $0.080 \lambda_{\mathrm{L}}$. The structure is made up of four layers. The top is metallic bow-tie structure with lumped resistances. The second one is $0.5 \mathrm{~mm}$-thick dielectric substrate with a relative dielectric constant of 4.4 and the loss tangent of 0.02 , respectively. The thickness of the air layer between substrate and the ground metallic layer is $10 \mathrm{~mm}$. The two-dimensional structure of the WBMA is presented in Fig. 1(b). To obtain wider band absorption and higher absorptivity, all the parameters of the unit cell are optimized using a High Frequency Structure Simulator (Ansoft HFSS.14.0). Table 1 presents the optimized values of all the parameters. Because of axial symmetry of the structure, the absorption performance is insensitive to the polarization direction of incident waves.

\subsection{Equivalent Circuit Model and Impedance Analysis of the WBMA}

When EM wave is incident on the surface of WBMA, the absorptivity of WBMA can be described by $A(w)=$ $1-R(w)-T(w), R(w)=\left|S_{11}(w)\right|^{2}$ and $T(w)=\left|S_{21}(w)\right|^{2}$ are the reflection coefficient and transmission coefficient, respectively. Because the WBMA is backed with a metallic plane, the transmission coefficient is zero and the absorptivity can be determined as $A(w)=1-\left|S_{11}(w)\right|^{2}$. Figure 2 shows the simulated absorptivity spectra of the unit cell without and with lumped resistances for a normal incident wave. The absorptivity is about zero without loading resistances. However, the absorptivity characteristics are improved significantly after loading resistances. Simulated results show that the absorber exhibits absorptivity above $90 \%$ from $2.3 \mathrm{GHz}$ to $9.6 \mathrm{GHz}$ with a relative absorption bandwidth of about $123 \%$. Thus, it attains a wideband impedance match.

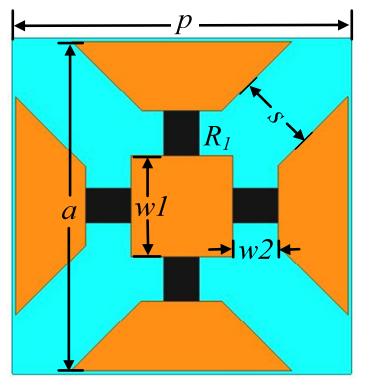

(a)

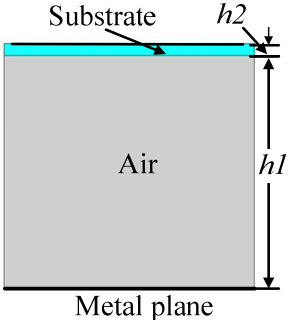

(b)
Fig. 1. Geometry of the WBMA unit cell: (a) the top view, (b) the side view.
Parameters

$w 1$

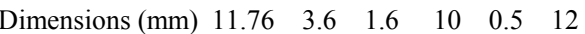

Tab. 1. Dimensions of the proposed WBMA unit cells.

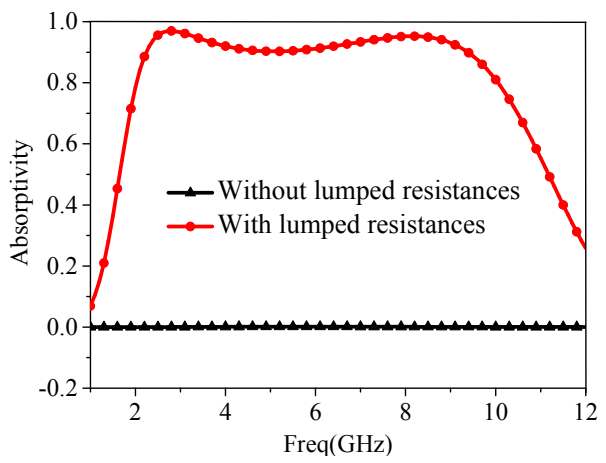

Fig. 2. Simulated absorptivity before and after loading the resistances.

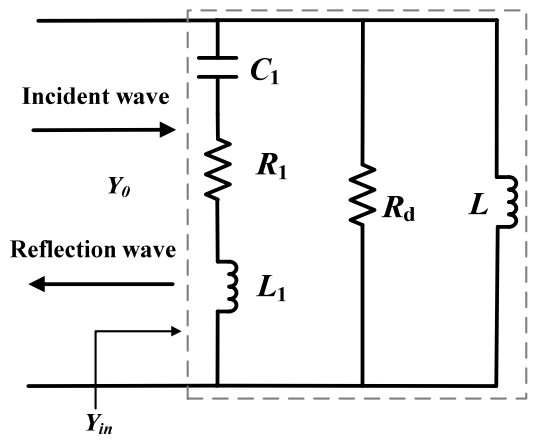

Fig. 3. The equivalent circuit model of the WBMA.

In order to explain the mechanism of wideband absorption, equivalent circuit model and impedance matching is analyzed. As Figure 3 presents, the proposed WBMA can be equivalent to a simplified circuit model using the transmission-line equivalent circuit theory. The slot between the metallic bow-tie structures is equivalent to capacitance $C_{1}$. The lumped resistance is described by resistance $R_{1}$. The metallic bow-tie structure and square patch can be depicted by inductance $L_{1}$. The loss of dielectric is equivalent to $R_{\mathrm{d}}$. And the backed metal ground equals to inductance $L$.

Firstly, the equivalent impedance of WBMA can be defined as follows:

$$
z(w)=\sqrt{\frac{\left[1+S_{11}(w)\right]^{2}-S_{21}(w)^{2}}{\left[1-S_{11}(w)\right]^{2}-S_{21}(w)^{2}}} .
$$

The relationship between the reflection coefficient and equivalent impedance can be described by:

$$
R(w)=\frac{z(w)-\eta_{0}}{z(w)+\eta_{0}} .
$$

From the view of equivalent circuit model, the effective input admittance of the equivalent circuit is expressed by: 


$$
Y_{\text {in }}(w)=\frac{1}{1 /\left(\mathrm{j} w C_{1}\right)+\mathrm{j} w L_{1}+R_{1}}+\frac{1}{R_{\mathrm{d}}}+\frac{1}{\mathrm{j} w L} .
$$

As we know, the impedance is inversely proportional to the admittance. Thus, the reflection coefficient can be described with equivalent admittance by:

$$
R(w)=\frac{z(w)-\eta_{0}}{z(w)+\eta_{0}}=\frac{1 / Y_{\text {in }}(w)-1 / Y_{0}}{1 / Y_{\text {in }}(w)+1 / Y_{0}}=\frac{Y_{0}-Y_{\text {in }}(w)}{Y_{0}+Y_{\text {in }}(w)} .
$$

The relative equivalent impedance is represented as:

$$
z_{\mathrm{e}}(w)=\frac{z(w)}{\eta_{0}}=\frac{Y_{0}}{Y_{\text {in }}(w)} .
$$

Thus, the reflection coefficient is simplified as:

$$
R(w)=\frac{z_{\mathrm{e}}(w)-1}{z_{\mathrm{e}}(w)+1} .
$$

The value of $R_{\mathrm{L}}$ is zero before loading lumped resistance. Figure 4(a) presents the relative equivalent impedance, and two inserted small pictures illustrate the enlarged details. The real part is always near zero. On the contrary, the imaginary part always deviates away from zero. It is obviously seen that there is a steep change at $4.8 \mathrm{GHz}$. In order to explain this phenomenon, the absorptivity is presented in Fig. 4(b). Though the absorptivity is low, a peak value at $5.2 \mathrm{GHz}$ exists owing to weak electromagnetic resonance of the metallic patches. According to (5), (6) and Fig. 4, we can get a conclusion that relative equivalent impedance of the unit without lumped resistance doesn't match the free-space impedance. It means that it is difficult to realize wideband absorption.

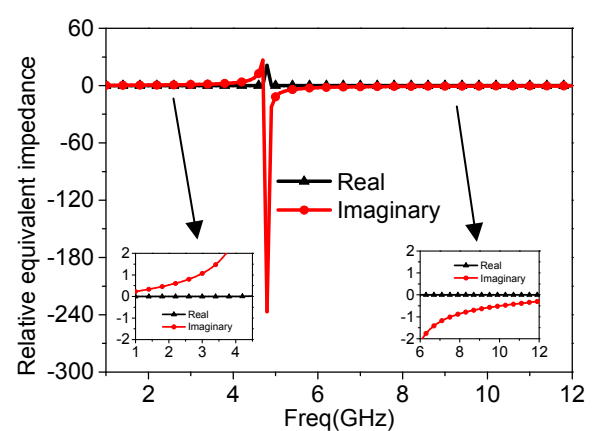

(a)

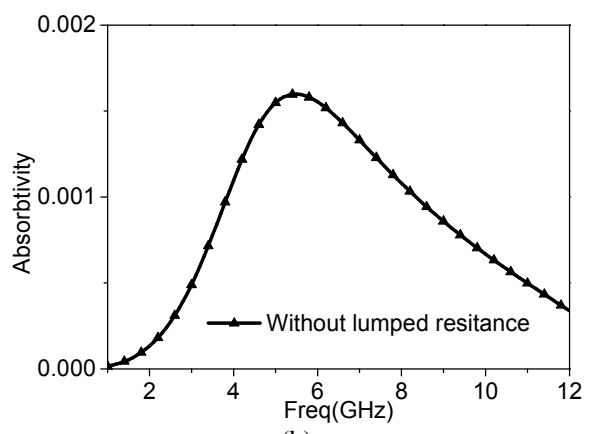

(b)

Fig. 4. The properties of the unit without lumped resistance: (a) relative equivalent impedance, (b) absorptivity.
After loading lumped resistance, the equivalent admittance has been changed, which causes matching to freespace $Y_{0}$. Then, the reflection coefficient is changed correspondingly based on (4). When the equivalent impedance of an absorber matches that of free-space, the reflection coefficient will approach zero, resulting in perfect absorption of the incident wave with no reflection. According to (6), if the reflection coefficient is about zero, the real part of the relative equivalent impedance must be close to unity, and the imaginary part should be around zero. As depicted in Fig. 5, the equivalent impedance of the WBMA approaches the wave impedance of free space in the frequency range from $2.3 \mathrm{GHz}$ to $9.6 \mathrm{GHz}$. It indicates that the wideband resonance is achieved owing to the introduction of lumped resistance.

The value of resistance is a key factor for impedance matching. Therefore, it is necessary to discuss the effect of it. Figure 6 shows the absorptivity with different resistance values. When resistance value is $50 \Omega$, the absorptivity of WBMA from $3.2 \mathrm{GHz}$ to $8 \mathrm{GHz}$ reveals deterioration. And for $120 \Omega$, good absorptivity is realized built on the sacrifice of absorption bandwidth. Therefore, $75 \Omega$ is chosen as the resistance value for overall consideration.

Figure 7 presents the equivalent circuit diagram of two adjacent units in the direction of y-axis intuitively. The metallic bow-tie structure and square patch are equivalent to $L_{10}$ and $L_{11}$, respectively. $L_{1}$ used to simplify in Fig. 3 is equal to $L_{10}$ plus $L_{11}$. And the value of $R_{1}$ is twice that of $R_{\mathrm{L}}$. Thus, the upper surface is equivalent to a RLC series circuit.

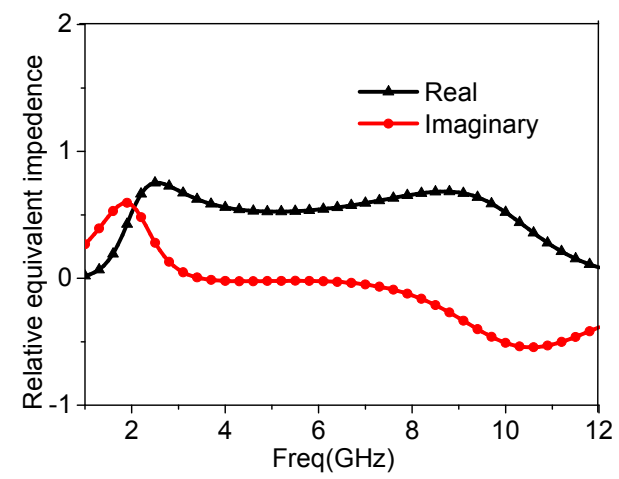

Fig. 5. Simulated relative equivalent impedance of the WBMA.

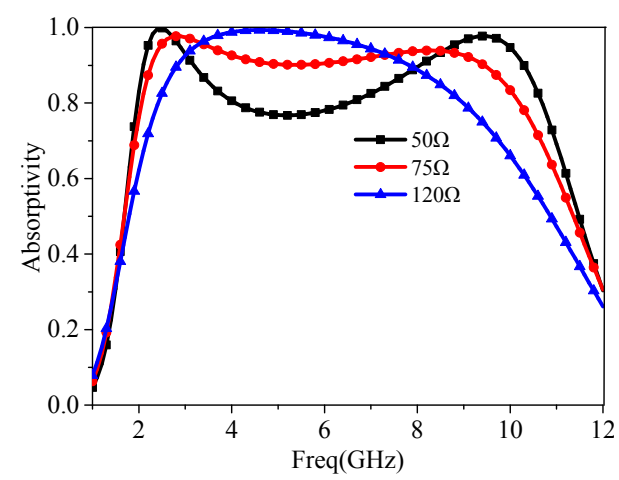

Fig. 6. Absorptivity with different resistance values. 


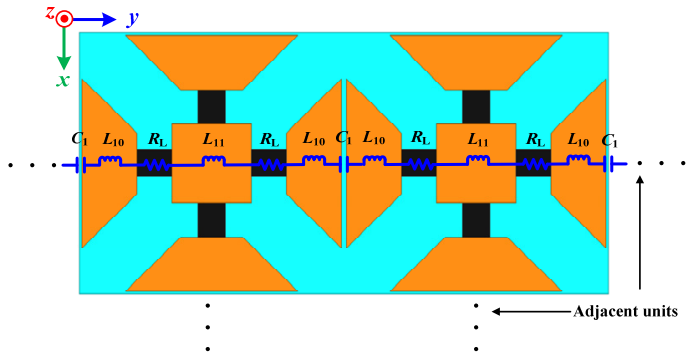

Fig. 7. The circuit diagram of the top surface.

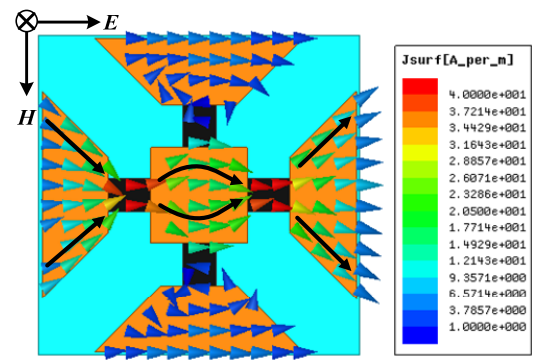

Fig. 8. The vector diagram of surface current distribution at $6 \mathrm{GHz}$.

The capacitance value is defined as presented in (7). $k$ and $\varepsilon$ are constant. The distance $d$ between the plates and the capacitor plate area $S$ are crucial to the value. The capacitance value is inversely proportional to the distance $d$. In contrast, it is proportional to the area $S$.

$$
C=\frac{\varepsilon S}{4 \pi k d} .
$$

In this paper, the distance $d$ between adjacent metallic bow-tie structures is very small. And the length of the edge is increased by using the butterfly gradient structure, which leads to an increase in the equivalent area $S$. Consequently, the equivalent capacitance $C_{1}$ is increased greatly. Furthermore, the vector diagram of surface current distribution at $6 \mathrm{GHz}$ is depicted in Fig. 8. Since the metallic bow-tie structure extends the path length of current along the edge of patch, the equivalent inductance $L_{10}$ is increased effectively. Therefore, the design of metallic bow-tie structure increases the equivalent capacitance and the equivalent inductance, making the absorber resonant at a lower frequency based on the approximate equation $f=1 /(2 \pi \sqrt{L C})$. Hence, it reduces the overall size.

\subsection{Surface E-field Distributions and Surface Current Distributions}

To further illustrate the effect of WBMA, the surface E-field distributions and surface current distributions are analyzed. The surface E-field distributions of the unit with and without resistances are depicted in Fig. 9. After loading resistances, the induced surface E-field is restrained at the edge of metallic bow-tie structure. Simultaneously, it is easily found that the surface E-field close to the edge of cell structure is also very strong in Fig. 10. It indicates this structure increases the equivalent capacitance and equiva- lent inductance so that it is beneficial for miniaturization. Figure 11 shows the surface current distribution for double mode of the cell structure. When EM waves enter into the structure, the induced surface current always increases greatly in the lumped resistances which are between the square metal and bow-tie metal patch. When induced current flowing the resistors, EM energy is lost in the form of thermal energy. Owing to the axial symmetry, it owns good absorption performance for arbitrary modes.

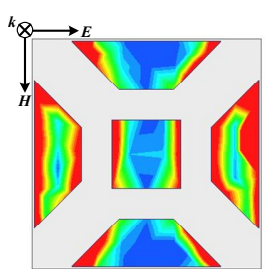

(a)

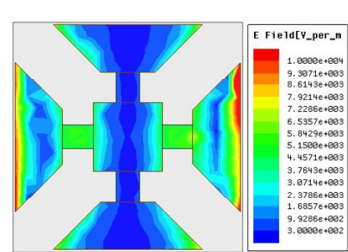

(b)
Fig. 9. Surface E-field distributions at $6 \mathrm{GHz}$ : (a) without lumped resistances; (b) with lumped resistances.

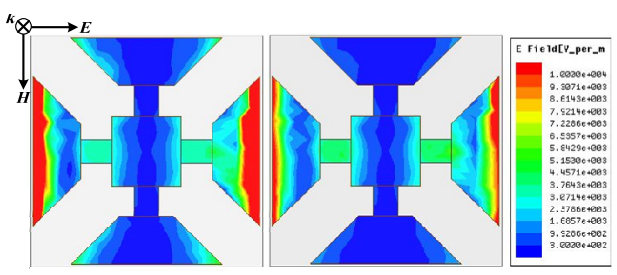

(a)

(b)

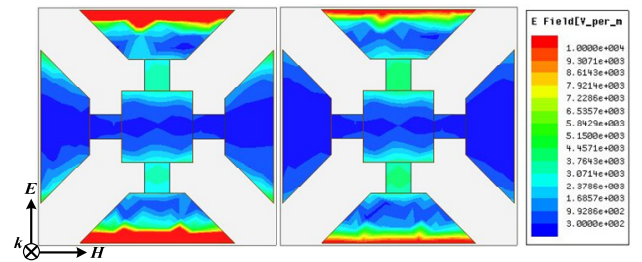

(c)

(d)

Fig. 10. Surface E-field distributions of WBMA under TE-polarized incident wave (a) at $4 \mathrm{GHz}$ and (b) at $8 \mathrm{GHz}$; under TM polarized incident wave (c) at $4 \mathrm{GHz}$ and (d) at $8 \mathrm{GHz}$

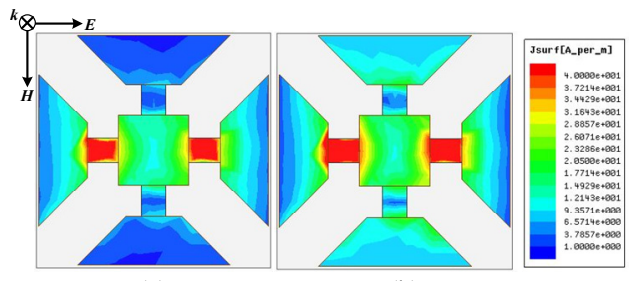

(a)

(b)

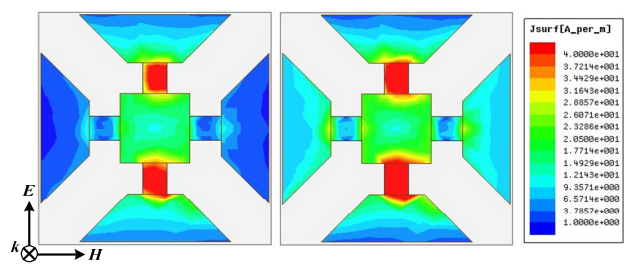

(c)

(d)

Fig. 11. Surface current distributions of WBMA under TE-polarized incident wave (a) at $4 \mathrm{GHz}$ and (b) at $8 \mathrm{GHz}$; under TM polarized incident wave (c) at $4 \mathrm{GHz}$ and (d) at $8 \mathrm{GHz}$. 


\subsection{Analysis of Different Parameters}

In order to obtain wideband impedance matching and structure miniaturization, the effect of air layer height and metal patterns length are analyzed. As shown in Fig. 12(a), different values of air layer height are investigated. As the height $h 1$ increases, the operation band shifts toward lower frequency and the absorptivity at the lowest frequency becomes better. However, the absorption performances at the highest frequency worsen with $h 1$ increases. When $a$ increases, the absorption performance at the highest frequency remains nearly unchanged, while that at the lowest frequency shifts toward lower frequency with a slight decrease in absorptivity. Thus, considering the absorptivity band and miniaturization, the optimal parameters are $h 1=10 \mathrm{~mm}$ and $a=11.76 \mathrm{~mm}$.

Figure 13 represents the absorptivity performance at normal and oblique incidence for TE and TM polarized waves. The absorptivity of TM polarization is better than that of TE polarization at oblique incidence. In order to illustrate the reason of this phenomenon, Figure 14 gives out the schematic diagram of electromagnetic wave at oblique incidence. The magnetic field is always parallel to the surface of WBMA under TE polarized wave. And the electric field is at an angle of $\theta$ from the normal direction of the surface. On the contrary, the electric field is always parallel to the surface of WBMA under TM polarized wave. Thus, it leads to different absorbance at oblique incidence under different polarized waves. Since both the magnetic field and electric field are parallel to the surface at normal incidence, the absorptivity of the WBMA under

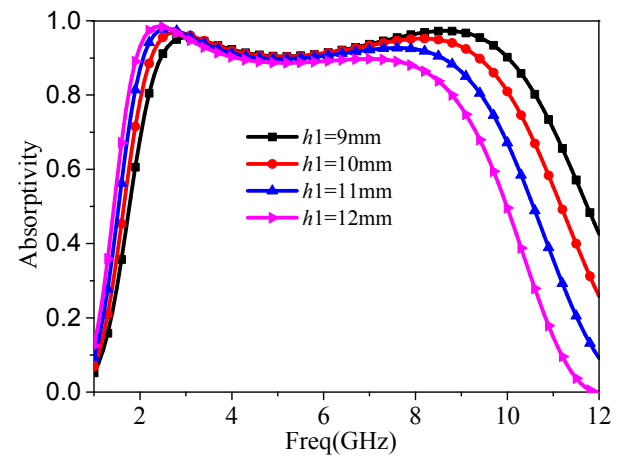

(a)

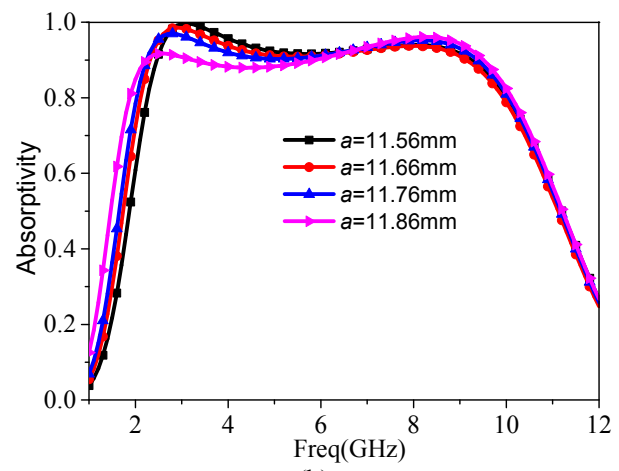

(b)

Fig. 12. Effect of various parameters in terms of absorptivity performance: (a) air height $h 1$;(b) metal patterns length $a$.

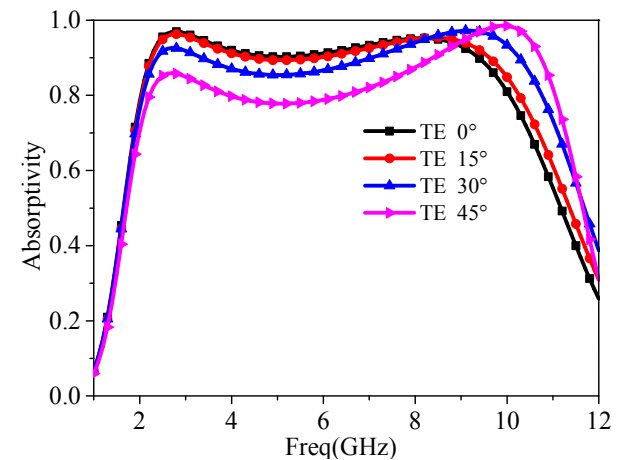

(a)

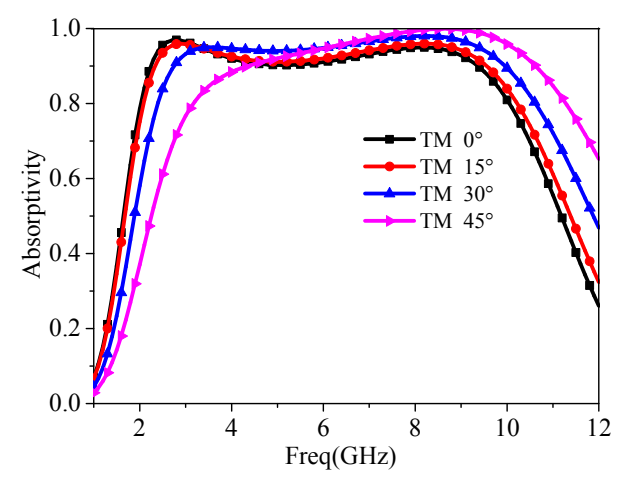

(b)

Fig. 13. Simulated absorptivity for different incidence angles at (a) TE polarized wave;(b) TM polarized wave.

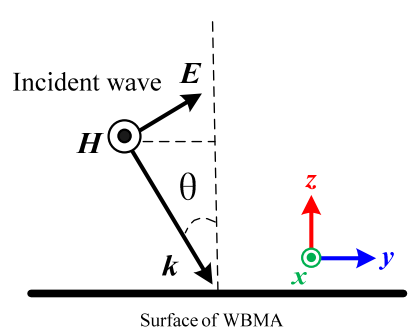

(a)

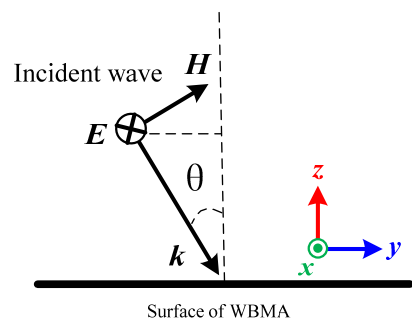

(b)
Fig. 14. The schematic diagram of electromagnetic wave at oblique incidence under (a) TE polarized wave and (b) TM polarized wave.

TE polarized wave is the same as the one under TM polarized wave. Even so, the WBMA also remains high absorptive at oblique incidence for both polarized waves.

Lastly, the properties and comparisons between different WBMA structures are presented, as shown in Tab. 2 . There is no doubt that the proposed WBMA has an advantage of miniaturization. Compared with other absorbers, its absorbing bandwidth also performs well.

\begin{tabular}{|c|c|c|c|c|}
\hline Absorber & $\begin{array}{c}\text { Bandwidth } \\
(\mathrm{GHz})\end{array}$ & $\begin{array}{c}\text { Fractional } \\
\text { bandwidth } \\
(\%)\end{array}$ & $\begin{array}{c}\text { Thickness } \\
\left(\lambda_{\mathrm{L}}\right)\end{array}$ & $\begin{array}{c}\text { Periodicity } \\
\left(\lambda_{\mathrm{L}}\right)\end{array}$ \\
\hline Ref. [14] & $4.0-18.0$ & 120 & 0.029 & 0.200 \\
\hline Ref. [15] & $7.0-37.4$ & 137 & 0.089 & 0.187 \\
\hline Ref. [16] & $1.9-7.3$ & 117 & 0.076 & 0.158 \\
\hline This paper & $2.3-9.6$ & 123 & 0.080 & 0.092 \\
\hline
\end{tabular}

Tab. 2. Comparison between different WBMAs. 


\section{Experiment Results}

Figure 15 shows an $18 \times 18$ array of the WBMA that we fabricated and tested to verify the absorptivity properties

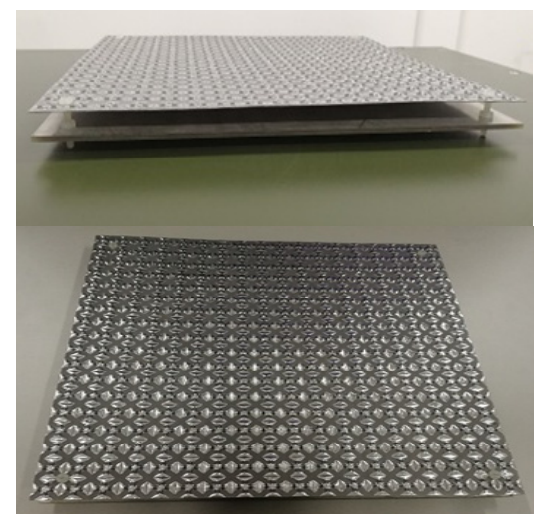

Fig. 15. Photograph of the WBMA sample.

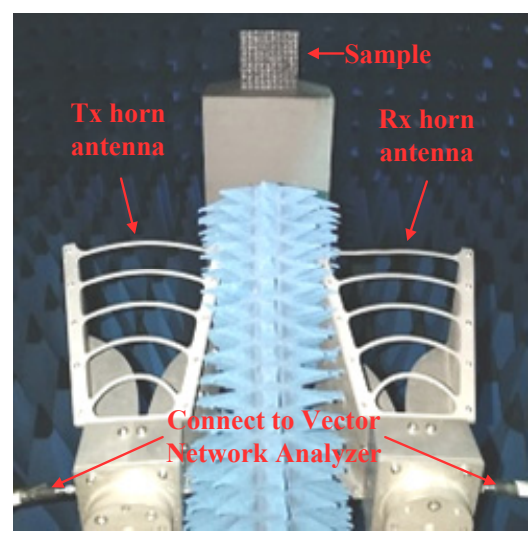

Fig. 16. Photograph of the experimental environment.

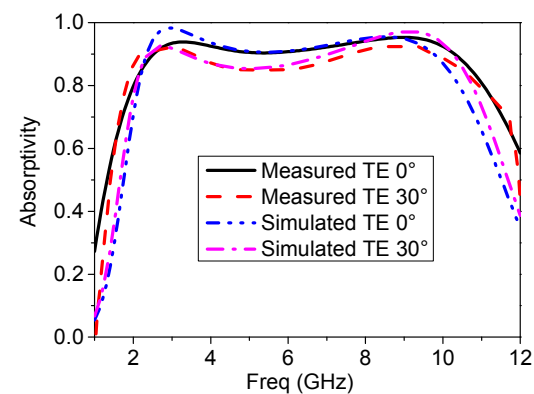

(a)

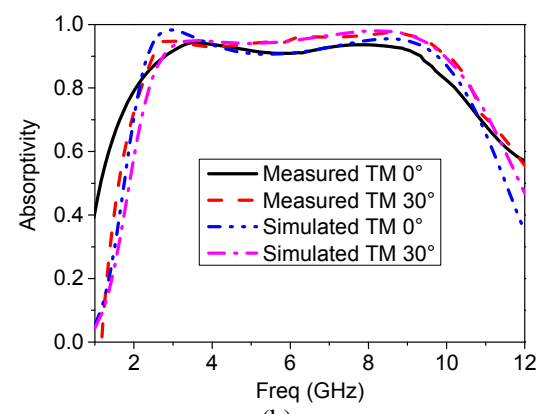

(b)

Fig. 17. Measured absorption response for (a) TE polarization and (b) TM polarization. of the simulated results. Measured results of reflection coefficient are obtained by Agilent 5230C network analyzer in an anechoic chamber, as shown in Fig. 16.

Figure 17 depicts the measured absorptivity performance at normal and oblique incidence for TE and TM polarized waves. We can conclude that the measured relative absorptivity bandwidth (greater than 90\%) is about $124 \%(2.2 \mathrm{GHz}-9.4 \mathrm{GHz})$ under normal incidence. And the measured results are almost in reasonable agreement with the simulations. In addition, because of the fabrication errors and the experimental environment, there exists an error between simulated and experimental results. In conclusion, the absorptivity of the prospered WBMA is verified by fabrication and measurement.

\section{Conclusion}

In this paper, a polarization and angular insensitive microwave absorber with miniaturized structure is designed, fabricated and measured. By analyzing impedance matching, surface E-field distributions and surface current distributions, the wideband operational mechanism is explained reasonably. The design of metallic bow-tie structure increases the equivalent capacitance and equivalent inductance, which helps realize miniaturization. The periodicity of unit is about $0.092 \lambda_{\mathrm{L}}$ at the operating lowest frequency, while the total thickness is only $0.080 \lambda_{\mathrm{L}}$. Simulated and experimental results show that the bandwidth of absorptivity more than $90 \%$ is from $2.3 \mathrm{GHz}$ to $9.6 \mathrm{GHz}$ with a relative absorption bandwidth of about $123 \%$.

\section{Acknowledgments}

This work was supported by the National Natural Science Foundation of China (Nos. 61471389, 61501494, 61671464 and 61701523), the Natural Science Foundational Research Fund of Shaanxi Province (No. 2017JM6025), and the Young Talent Fund of University Association for Science and Technology in Shaanxi, China (No. 20170107). Authors also thank the reviewers for their valuable comments.

\section{References}

[1] LANDY, N. I., SAJUYIGBE, S., MOCK, J. J., et al. Perfect metamaterial absorber. Physical Review Letters, 2008, vol. 100 , p. 1-4. DOI: 10.1103/PhysRevLett.100.207402

[2] LANDY, N. I., BINGHAM, C. M., TYLER, T., et al. Design, theory, and measurement of a polarization-insensitive absorber for terahertz imaging. Physical Review B, 2009, vol. 79, p. 1-6. DOI: 10.1103/PhysRevB.79.125104

[3] GHOSH, S., BHATTACHARYYA, S., CHAURASIYA, D., et al. Polarization-insensitive and wide-angle multi-layer metamaterial absorber with variable bandwidths. Electronics Letters, 2015, vol. 51, no. 14 , p. 1050-1052. DOI: 10.1049/el.2015.1167 
[4] ZHONG, H. T., YANG, X. X., CHONG TAN, et al. Triple-band polarization-insensitive and wide-angle metamaterial array for electromagnetic energy harvesting. Applied Physics Letters, 2016, vol. 109 , p. 1-4, article no. 253904. DOI: $10.1063 / 1.4973282$

[5] SHANG, S., YANG, S. Z., LU TAO., et al. Ultrathin triple-band polarization-insensitive wide-angle compact metamaterial absorber. American Institute of Physics Advances, 2016, vol. 6, p. $1-8$, article no. 075203 . DOI: $10.1063 / 1.4958660$

[6] LI, H., YUAN, L. H., ZHOU, B., et al. Ultrathin multiband gigahertz metamaterial absorbers. Journal of Applied Physics, 2016, vol. 110, p. 1-8. DOI: 10.1063/1.3608246

[7] AN, S. N., XU, H. B., ZHANG, Y. L., et al. Design of a polarization-insensitive wideband tunable metamaterial absorber based on split semi-circle ring resonators. Journal of Applied Physics, 2017, vol. 122, p. 1-5, article no. 025113. DOI: 10.1063/1.4993717

[8] LI, W. C., ZHOU, X., YING, Y., et al. Polarization-insensitive wide-angle multiband metamaterial absorber with a double-layer modified electric ring resonator array. American Institute of Physics Advances, 2015, vol. 5, p. 1-9, article no. 067151. DOI: $10.1063 / 1.4923194$

[9] ZENG, X. L., ZHANG, L. X., WAN, G. B., et al. Active metamaterial absorber with controllable polarization and frequency. Electronics Letters, 2017, vol. 53, no. 16, p. 1085-1086. DOI: 10.1049/el.2017.1618

[10] YUAN, H., ZHU, B. O., FENG, Y. A frequency and bandwidth tunable metamaterial absorber in X-band. Journal of Applied Physics, 2015, vol. 117, p. 1-6, article no. 173103. DOI: $10.1063 / 1.4919753$

[11] HE, Y., JIANG, J. J., MI, C., et al. Design of an adjustable polarization-independent and wideband electromagnetic absorber. Journal of Applied Physics, 2016, vol. 119, p. 1-5, article no. 105103 . DOI: $10.1063 / 1.4943593$

[12] LI, S., CAO, X., GAO, J., et al. Fractal metamaterial absorber with three-order oblique cross dipole slot structure and its application for in-band RCS reduction of array antennas. Radioengineering, 2014, vol. 23, no. 4, p. 1048-1054. ISSN: 1210-25-12

[13] PAN, W., YU, X., ZHANG, J., et al. A novel design of broadband terahertz metamaterial absorber based on nested circle rings. IEEE Photonics Technology Letters, 2016, vol. 28, no. 21, p. 2335-2338. DOI: 10.1109/LPT.2016.2593699

[14] YUAN, W., CHEN, Q., XU, Y. S., et al. Broadband microwave absorption properties of ultrathin composites containing edge-split square-loop FSS embedded in magnetic sheets. IEEE Antennas and Wireless Propagation Letters, 2017, vol. 16, p. 278-281. DOI: 10.1109/LAWP.2016.2572734

[15] LI, L., LV, Z. Y. Ultra-wideband polarization-insensitive and wide-angle thin absorber based on resistive metasurfaces with three resonant modes. Journal of Applied Physics, 2017, vol. 122, p. 1-6, article no. 055104. DOI: 10.1063/1.4997468

[16] HAN, Y., CHE, W. Q. Low-profile broadband absorbers based on capacitive surfaces. IEEE Antennas and Wireless Propagation Letters, 2017, vol. 16, p. 74-78. DOI: 10.1109/LAWP.2016.2556753

\section{About the Authors...}

Junxiang LAN was born in Xinyang, Henan province in 1994. He received the B.S. degree from the Northwest University, China in 2016. He currently works towards the M.S. degree at the Information and Navigation College.
His research interests mainly include the design of wideband microwave absorber and its application on antennas.

Xiangyu CAO received the B. Sc and M.A. Sc degrees from the Air Force Missile Institute in 1986 and 1989, respectively. She joined the Air Force Missile Institute in 1989 as an assistant teacher. She became an associate professor in 1996. She received Ph.D. degree from the Missile Institute of Air Force Engineering University in 1999. From 1999 to 2002, she was engaged in postdoctoral research in Xidian University, China. She was a Senior Research Associate in the Department of Electronic Engineering, City University of Hong Kong from Jun. 2002 to Dec. 2003. She is currently a professor of Air Force Engineering University of CPLA. Her research interests include computational electromagnetic, smart antennas, electromagnetic metamaterial and their antenna applications, and electromagnetic compatibility. She is a reviewer of Applied Physics Letter, Journal of Applied Physics, IEEE Transactions on Antennas \& Propagation, and IEEE Antennas Wireless Propagation Letter.

Jun GAO received the B. Sc and M. A. Sc degrees from the Air Force Missile Institute in 1984 and 1987, respectively. He joined the Air Force Missile Institute in 1987 as an assistant teacher. He became an associate professor in 2000. He is currently a professor of the Information and Navigation College, Air Force Engineering University of CPLA. He has authored and coauthored more than 100 technical journal articles and conference papers, and holds one China soft patent. His research interests include smart antennas, electromagnetic metamaterials and their antenna applications.

Lili CONG received her M.S. degree from AFEU in 2016. She is currently working towards the Ph.D. degree. Her research interests include microwave absorber and electromagnetic scattering theory.

Siming WANG received B.S. from the Air Force Engineering University, Xi'an China, in 2016. She is currently working toward M.S. degree at the Information and Navigation College of the Air Force Engineering University. She has been working with the Military Communication and Navigation Antenna and EMC Lab, since 2016. Her main interests include metasurface antennas design, antenna array and RCS reduction.

Huanhuan YANG has received the M. Eng. degree and Ph.D. degree in Electronic Science and Technology (Electronic Field and Microwave Technology) at the Information and Navigation Institute, Air Force Engineering University, Xi'an, China, in 2012 and 2016, respectively. $\mathrm{He}$ is currently working as an instructor in the Information and Navigation Institute, Air Force Engineering University. His research activity has been focused in the broadband perfect microwave absorber, polarization rotation metasurface, and their application for RCS reduction of antennas. He has authored and coauthored more than 40 scientific papers in major journals and international conferences. 